\title{
Retinol Binding Protein-4 Circulating Levels Were Higher in Nonalcoholic Fatty Liver Disease Vs. Histologically Normal Liver From Morbidly Obese Women
}

\author{
Ximena Terra ${ }^{1}$, Teresa Auguet ${ }^{1,2}$, Montserrat Broch ${ }^{1}$, Fàtima Sabench ${ }^{3}$, Mercè Hernández $^{3}$, \\ Rosa M. Pastor ${ }^{4}$, Isabel M. Quesada ${ }^{1}$, Anna Luna ${ }^{1}$, Carmen Aguilar ${ }^{1}$, Daniel del Castillo ${ }^{3}$ and \\ Cristóbal Richart ${ }^{1,2}$
}

\begin{abstract}
We aimed to analyze the retinol binding protein-4 (RBP4) messenger RNA (mRNA) expression profiles in adipose tissues and liver of morbidly obese (MO) women with or without nonalcoholic fatty liver disease (NAFLD), and to study the relationships with other pro- and anti-inflammatory adipokines in vivo and in vitro. We performed a cross-sectional analysis of subcutaneous adipose tissue (SAT), visceral adipose tissue (VAT) and liver samples from four lean and 45 MO women with or without NAFLD by enzyme-linked immunosorbent assay and real-time reverse transcription-PCR. We also studied RBP4 expression in HepG2 hepatocytes under various inflammatory stimuli. Circulating RBP4 levels were higher in MO women, and specifically, in MO subjects with NAFLD compared with normal liver controls (lean and MO). RBP4 liver expression was higher in nonalcoholic steatohepatitis (NASH)-moderate/severe than in NASHmild. Overall RBP4 gene expression was higher in liver than in adipose tissues. Among them, the higher expression corresponded to SAT. VAT expression was lower in the MO cohort. In HepG2, RBP4 mRNA expression was reduced by tumor necrosis factor (TNF)- $\alpha$ and increased by adiponectin treatment. In conclusion, the results obtained in MO women with NAFLD, brings up the use of RBP4 and other adipokines as a panel of noninvasive molecular biomarkers when NAFLD is suspected. Further studies are needed with other obesity groups.
\end{abstract}

\section{INTRODUCTION}

Nonalcoholic fatty liver disease (NAFLD) has frequently been associated with obesity, type 2 diabetes mellitus (T2DM), hyperlipidemia, and insulin resistance, which are the main features of metabolic syndrome $(1,2)$. NAFLD is an increasingly recognized condition that has become the most common liver disorder in the developed world (1). NAFLD causes a wide spectrum of liver damage, ranging from simple steatosis (SS) to cirrhosis. In most cases, SS does not progress to a more severe disease, but $\sim 20-30 \%$ of patients have histological signs of fibrosis and necroinflammation, indicating a presence of nonalcoholic steatohepatitis (NASH) (3). The pathogenesis of NAFLD is poorly understood and much of the current opinion remains hypothetical.

Obesity is considered an important risk factor, though not all patients with NAFLD are obese (4). In obesity, an altered secretion of proteins takes place in the adipose tissue, the so-called adipokines, which have wide-ranging endocrine and paracrine effects on general metabolism (5). These adipokines are considered important determinants of obesity-related disorders such as insulin resistance, inflammation, and dyslipidemia (6). Consequently, there is growing evidence that adipokines are one of the causal links between obesity and NAFLD (7).

A recently identified adipokine is the retinol binding protein-4 (RBP4), the specific carrier protein of retinol (vitamin A) in the blood. Although the major tissue site of RBP4 synthesis in the body is the liver, other sites of synthesis have been reported such as adipose tissues, kidney, and brain (8-10). It has been put forward that adipose tissue in humans, as occurs in mice, has the second highest level of RBP4 expression (8).

Circulating RBP4 levels have been associated with estimations of adiposity and insulin resistance (11-13). Interestingly, we and others have reported a downregulation of RBP4 messenger RNA (mRNA) in subcutaneous adipose tissue (SAT) 
and an association of blood RBP4 with lipid parameters in obesity $(14,15)$. In addition, since RBP4 was more related to liver fat than to abdominal fat depots, this adipokine has been suggested to contribute to an increase in fat accumulation in the liver (16).

RBP4 has scarcely been studied in patients with SS and NASH lesions and has had discordant results. Two studies using ultrasonography and magnetic spectroscopy found high systemic levels of RBP4 in subjects with NAFLD $(17,18)$, but no correlation was found between systemic RBP4 levels and the histological grade of steatosis or necroinflammation in three biopsy-controlled studies (19-21).

To our knowledge, no study has yet analyzed simultaneously the levels of RBP4 in blood and its mRNA expression in subcutaneous and visceral fat depots and in liver. In the present study, using the above approach, we aimed (i) to find out the gene expression profiles of RBP4 in both SAT and visceral adipose tissue (VAT), and in the liver of morbidly obese (MO) women with or without NAFLD, (ii) to analyze if systemic RBP4 might be related to its expression in any of the above tissues, and (iii) to study the relationships of this adipokine with other adipokines and with the NAFLD histological stage.

\section{METHODS AND PROCEDURES \\ Subjects}

In this study, we analyzed serum RBP4 levels in 49 Spanish women of European descent: four lean $\left(\mathrm{BMI}<25 \mathrm{~kg} / \mathrm{m}^{2}\right)$ and $45 \mathrm{MO}$ women $\left(\mathrm{BMI}>40 \mathrm{~kg} / \mathrm{m}^{2}\right)$. The liver pathology was analyzed in lean controls and in MO women. Among the obese patients, we found 11 with normal liver (NL) histology and 34 with NAFLD. The diagnosis of NAFLD was made by the following criteria: (i) liver pathology, (ii) an intake of $<10 \mathrm{~g}$ of ethanol/day, and (iii) appropriate exclusion of other liver diseases. Liver samples were stained with hematoxylin and eosin and Masson's trichrome stains and scored by an experienced hepatopathologist using a modification of the methods proposed by Brunt et al. (22) and Kleiner et al. (23). According to the liver pathology and their BMI, patients were subclassified into the following groups: (i) controls: lean patients with $\mathrm{NL}$ histology (BMI $\left.<25 \mathrm{~kg} / \mathrm{m}^{2}, n=4\right)$, (ii) MO with normal liver histology (BMI $>40 \mathrm{~kg} / \mathrm{m}^{2}, n=11$ ), (iii) MO with SS (BMI $>40 \mathrm{~kg} / \mathrm{m}^{2}$, micro/ macrovesicular steatosis without inflammation or fibrosis, $n=13$ ), (iv) MO with NASH (BMI $>40 \mathrm{~kg} / \mathrm{m}^{2}$, Brunt grade $1-3, n=21$ ).

Liver biopsies were taken from MO patients if the clinical and biochemical criteria in successive analyses indicated possible inflammatory activity in the liver. Furthermore, experts using laparoscopic techniques needed to have macroscopic fatty liver abnormalities observed during surgery. The liver biopsy was carried out only on those patients who fulfilled both of these criteria and/or fibrosis, which established the presence of possible inflammatory liver. The Hospital's Pathology Service ensured on informed consent in order to use the liver samples of the control group and, once NL histology had been diagnosed, the minimum excess sample was used to carry out gene expression analysis. In the control group, the biopsies had been taken to aid diagnosis by excluding any liver pathology.

Blood samples were collected with glass vacuum tubes without additives. The samples were processed and stored at $-80^{\circ} \mathrm{C}$ immediately.

Adipose tissue samples were collected from $\mathrm{MO}$ women who underwent bariatric surgery by laparoscopic gastric bypass and from lean patients who underwent laparoscopic cholecystectomy for benign gallbladder disease or laparoscopic hiatal hernia repair. SAT biopsies were taken from the right hypochondrion region and VAT biopsies were taken from the greater epiploon region. The same specialist collected samples from both locations. $\mathrm{MO}$ and controls were age-matched. All subjects had a stable weight with no fluctuations of more than $2 \%$ of their body weight for at least 3 months before surgery. Patients were excluded from the study if they had an acute illness, any current evidence of acute or chronic inflammatory or infective diseases, an abnormal renal clearance or any end-stage malignant diseases.

In the MO group, 10 women had T2DM; a diagnosis based on World Health Organization criteria (24). None of them were receiving insulin or on medication likely to influence endogenous insulin levels. There were no patients receiving hypolipemiant treatment.

The institutional review board approved the study. All participants gave written informed consent for participation in medical research.

\section{Biochemical analysis}

We determined the anthropometrical and metabolic features. Body height and weight were measured with the patient standing in light clothes and shoeless. BMI was calculated as body weight divided by squared height $\left(\mathrm{kg} / \mathrm{m}^{2}\right)$. The subjects' waist was measured with a soft tape midway between the lowest rib and the iliac crest. Laboratory studies included glucose, insulin, C-peptide, glycated hemoglobin ( $\left.\mathrm{HbA}_{1 c}\right)$, total cholesterol, high-density lipoprotein cholesterol, triglycerides, and transaminases, all of which were analyzed using a conventional automated analyzer. Insulin resistance (IR) was estimated using homeostasis model assessment of IR (HOMA-IR).

RBP4 in serum samples were measured by nephelometry (Siemens Healthcare Care Diagnostics products GmbH, Marburg, Germany). Intra- and inter-assay coefficients of variation were 1.8 and $1.8 \%$, respectively. No cross-reactivity of the applied antibody is known. The assay was approved for in vitro diagnostic use in the United States and Europe and demonstrated to show excellent correlations with results obtained from radial immunodiffusion. Adiponectin (Linco Research, St Charles, MO), TNFR2 (AssayPro, St Charles, MO), and resistin (Biovendor, Modrice, Czech Republic) were measured in duplicate using enzyme-linked immunosorbent assays following the manufacturers' instructions.

\section{HepG2 cell culture and treatments}

HepG2 cells (ECACC cat no. 85017430) were grown in Dulbecco's modified Eagle medium (DMEM BE12-604E; Lonza, Barcelona, Spain) supplemented with $10 \%$ fetal bovine serum (Lonza), $25 \mathrm{mmol} / \mathrm{l} \mathrm{HEPES}$ (Lonza), $100 \mathrm{U} / \mathrm{ml}$ Penicillin/Streptomycin (Lonza), and 1\% nonessential amino acids (Invitrogen). Cells were incubated at $37^{\circ} \mathrm{C}$ in a humidified, $5 \% \mathrm{CO}_{2}$-enriched atmosphere and routinely split upon reaching $80-90 \%$ confluence. For treatments, cultures at $80-90 \%$ confluence were subcultured in growth medium at a density of $4 \times 10^{5} \mathrm{cells} / \mathrm{ml}$, and $1 \mathrm{ml}$ aliquots were seeded per well in 12-well plates (Greiner bio-one, Madrid, Spain). When cultures reached $80-90 \%$ confluence, growth medium was replaced with $1 \mathrm{ml}$ of fresh growth medium without fetal bovine serum containing $0,10,25,50,100,200 \mathrm{ng} / \mathrm{ml}$ tumor necrosis factor (TNF)- $\alpha$ or $0,50,100,500,1,000,2,000 \mathrm{ng} / \mathrm{ml}$ adiponectin (PROSPEC, Rehout, Israel). Cultured cells were lysed and collected after $48 \mathrm{~h}$ for subsequent mRNA expression analysis.

\section{RNA isolation and real-time PCR}

Total RNA from liver and VAT or cultured cells was isolated according to the manufacturers' protocols RNeasy mini kit or RNeasy midi kit, respectively (Qiagen, Barcelona, Spain). RNA was digested with DNase I (RNase-Free DNase set; Qiagen). First-strand cDNA was synthesized using an equal amount of total RNA with High Capacity RNA-to-cDNA Kit (Applied Biosystems, Madrid, Spain). The realtime quantitative PCR was carried out in a final volume of $20 \mu$, which contained $10 \mathrm{ng}$ of reverse-transcribed cDNA, $10 \mu \mathrm{l}$ of $2 \times$ TaqMan Fast Universal PCR Master Mix (Applied Biosystems) and $1 \mu \mathrm{l}$ TaqMan Assay predesigned by Applied Biosystems for the detection of RBP4, adiponectin, adiponectin receptor 2 , TNF- $\alpha$, resistin, and for GAPDH that was used as housekeeping gene. All reactions were carried out in triplicate in 96-well plates using the 7900HT Fast Real-Time PCR systems (Applied Biosystems). 


\section{Statistical analysis}

All the values reported are expressed as mean \pm s.d. and were analyzed using SPSS/PC+ for windows statistical package (v.15.0; SPSS, Chicago, IL). Differences between groups were calculated using the Student's $t$-test or one-way ANOVA analysis. The strength of association between variables was calculated using Pearson's method for parametric variables and Spearman's $\rho$-correlation test for nonparametric contrasts. $P$ values $<0.05$ were considered to be statistically significant.

\section{RESULTS}

\section{Patient's characteristics}

Patients' baseline characteristics, given in Table 1, show the mean and s.d. of the variables of interest. Patients were separated into control subjects $\left(\mathrm{C}, n=4, \mathrm{BMI}<25 \mathrm{~kg} / \mathrm{m}^{2}\right)$, and $\mathrm{MO}$ $\left(n=45, \mathrm{BMI}>40 \mathrm{~kg} / \mathrm{m}^{2}\right)$. MO subjects were further subclassified according to the liver histology: NL $(n=11)$, SS ( $n=$ $13)$, and NASH $(n=21)$. The groups were well matched for age. Compared with control group, MO groups displayed significantly higher values of fasting glucose, fasting insulin, HOMA-IR index, triglycerides, and low-density lipoprotein (LDL) cholesterol, although no differences were found between subgroups. We also determined the transaminase levels in MO women vs. lean controls. Among MO women, we found that those with SS or NASH showed significantly higher values of liver enzymes aspartate aminotransferase (AST), alanine aminotransferase (ALT), and $\gamma$-glutamyltraspeptidase (GGT) compared with those with NL (Table 1).
Table 1 also shows the circulating levels of adiponectin, resistin, and TNFR2. Results indicate that the circulating levels of TNFR2 were higher in the MO groups with NAFLD compared with NL, whereas adiponectin levels were lower in these groups. The analysis of the adipocytokine levels between SS and NASH showed that there were no differences between these groups.

\section{Circulating RBP4 levels}

RBP4 levels were significantly higher in MO women (C: $2.5 \pm$ 0.2 ; MO: $3.3 \pm 0.2 ; P=0.037)$. Furthermore, among MO subjects, those with NAFLD (SS and NASH) had higher levels of RBP4 compared both with controls and with those with NL (Table 1). Comparison between SS and NASH groups showed no significant differences $(P=0.237)$. Analysis within NASH patients did not show a relationship between concentrations of RBP4 and the degree of NASH (3.1 \pm 0.8 (mild) vs. $3.3 \pm 0.8$ (moderate/severe), $P=0.410$ ).

In the MO group, we found significant associations between RBP4 levels and triglycerides $(r=0.405, P=0.009)$, AST $(r=0.440, P=$ $0.010)$, and ALT ( $r=0.440, P=0.009)$. Furthermore, RBP4 in the circulation was negatively related with resistin levels $(r=-0.398, P$ $=0.010)$. By contrast, RBP4 levels were not correlated with BMI ( $r=$ $0.215, P=0.177)$ or HOMA-IR $(r=-0.040, P=0.810)$.

In the MO cohort, only triglyceride levels appeared as an independent factor that explained systemic concentrations of

Table 1 Baseline characteristics of the study cohort

\begin{tabular}{|c|c|c|c|c|}
\hline \multirow[b]{2}{*}{ Group } & \multirow{2}{*}{$\begin{array}{c}\text { Lean } \\
\text { Normal liver }(n=4)\end{array}$} & \multicolumn{3}{|c|}{ Morbid obese } \\
\hline & & Normal liver $(n=11)$ & Simple steatosis $(n=13)$ & $\mathrm{NASH}(n=21)$ \\
\hline Age (years) & $50.2 \pm 4.1$ & $47.2 \pm 1.7$ & $44.9 \pm 3.2$ & $47.0 \pm 2.3$ \\
\hline $\mathrm{BMI}\left(\mathrm{kg} / \mathrm{m}^{2}\right)$ & $23.1 \pm 0.7$ & $49.2 \pm 1.7^{a}$ & $49.5 \pm 2.2^{a}$ & $47.9 \pm 1.2^{a}$ \\
\hline Waist (cm) & $80.3 \pm 7.5$ & $134.3 \pm 5.1^{\mathrm{a}}$ & $127.6 \pm 1.8^{a}$ & $134.5 \pm 3.8^{a}$ \\
\hline Fasting glucose (mg/dl) & $105.0 \pm 5.3$ & $117.8 \pm 12.7$ & $120.5 \pm 7.4^{\mathrm{a}}$ & $127.1 \pm 8.4^{\mathrm{a}}$ \\
\hline Fasting insulin $(\mu \mathrm{UI} / \mathrm{I})$ & $8.0 \pm 1.9$ & $20.6 \pm 3.4^{a}$ & $20.2 \pm 3.2^{a}$ & $23.4 \pm 5.4^{a}$ \\
\hline $\mathrm{HbA}_{1 \mathrm{c}}(\%)$ & $4.5 \pm 0.1$ & $4.9 \pm 0.2$ & $5.7 \pm 0.3$ & $5.7 \pm 0.3$ \\
\hline HOMA-IRI & $2.1 \pm 0.6$ & $6.6 \pm 1.7^{a}$ & $5.7 \pm 0.9^{a}$ & $8.3 \pm 2.5^{a}$ \\
\hline Triglycerides (mg/dl) & $131.0 \pm 23.4$ & $155.2 \pm 12.6^{a}$ & $179.6 \pm 21.6^{a}$ & $183.4 \pm 16.8^{a}$ \\
\hline HDL-cholesterol (mg/dl) & $54.4 \pm 6.6$ & $39.1 \pm 2.6^{a}$ & $40.5 \pm 2.6^{a}$ & $40.0 \pm 1.5^{a}$ \\
\hline AST (IU/I) & $31.7 \pm 13.9$ & $28.5 \pm 8.3$ & $58.8 \pm 4.2^{a}$ & $48.9 \pm 5.3^{a}$ \\
\hline ALT (IU/I) & $30.7 \pm 21.9$ & $27.9 \pm 5.6$ & $64.3 \pm 6.2^{a}$ & $50.7 \pm 7.4^{a}$ \\
\hline GGT (IU/I) & $21.1 \pm 7.1$ & $17.2 \pm 2.3$ & $31.6 \pm 2.4^{a}$ & $29.5 \pm 3.3^{a}$ \\
\hline \multicolumn{5}{|l|}{ Adipocytokines } \\
\hline RBP4 (mg/dl) & $2.5 \pm 0.2$ & $2.7 \pm 0.2$ & $3.8 \pm 0.4^{a, b}$ & $3.3 \pm 0.2^{a, b}$ \\
\hline Adiponectin ( $\mu \mathrm{g} / \mathrm{ml})$ & $11.5 \pm 1.7$ & $8.8 \pm 0.7$ & $7.2 \pm 1.0^{\mathrm{a}}$ & $7.1 \pm 0.6^{a}$ \\
\hline TNFR2 (ng/ml) & $3.6 \pm 0.6$ & $3.9 \pm 0.5$ & $6.0 \pm 0.7^{a, b}$ & $5.5 \pm 0.3^{a, b}$ \\
\hline Resistin (ng/ml) & $4.0 \pm 0.5$ & $5.0 \pm 0.5$ & $4.4 \pm 0.5$ & $4.4 \pm 0.3$ \\
\hline \multicolumn{5}{|c|}{ 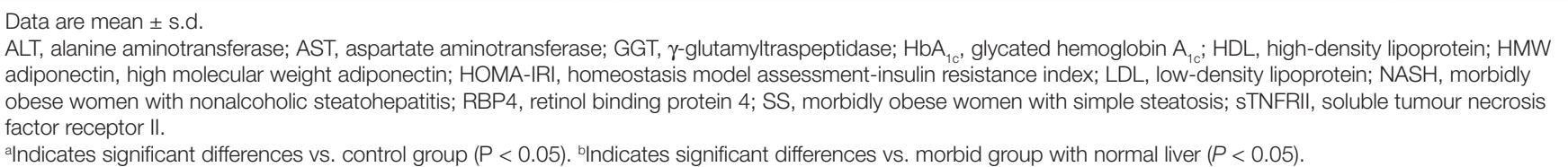 } \\
\hline
\end{tabular}


RBP4 ( $\beta=0.01, P=0.006,95 \%$ confidence interval (CI) $0.004-$ 0.02 ). The model had a $R^{2}$ of $39 \%$ and included triglycerides, AST, ALT, and GGT parameters.

Interestingly, when we analyzed the associations only in the group of women with NAFLD and after a multiple regression analysis, the level of triglycerides was the only parameter that alone could explain the RBP4 systemic concentrations $(\beta=$ $0.02, P=0.01,95 \%$ CI $\left.0.003-0.02, R^{2}=30 \%\right)$.

\section{Tissue-specific RBP4 and adiponectin gene expression} RBP4 and adiponectin mRNA SAT expression. RBP4 expression in SAT was higher in MO compared with lean subjects (Figure 1a). We observed similar RBP4 gene expression values in SAT between the four cohorts studied: C group, MO with NL, with SS or NASH (not significant $P$ value) (Figure 1d). Furthermore, we analyzed RBP4 expression between NASH-mild and NASH-moderate/severe groups. RBP4 was similarly expressed in both groups $(2.0 \pm 0.9$ vs. $3.3 \pm 2.4, P=0.35)$.

Adiponectin expression in SAT was significantly lower in the MO cohort compared with controls, but with no differences between MO subgroups (C: $9.5 \pm 1.4$ vs. MO: $4.0 \pm 0.3, P=$ $0.001)$. RBP4 and adiponectin gene expression in SAT were not correlated $(r=-0.381, P=0.099)$.
RBP4 mRNA VAT expression. Expression of RBP4 in VAT was significantly lower in $\mathrm{MO}$ women compared with the $\mathrm{C}$ group $(P=0.001)$ (Figure 1b). Among MO women, values were similar between women with NL and with SS or NASH $(P=0.68)$, (Figure 1e). In addition, there were no differences between NASH-mild group and NASH-moderate/severe group $(2.0 \pm 1.0$ vs. $1.6 \pm 0.6, P=0.51)$.

Adiponectin expression levels in VAT did not show differences between controls and MO groups (C: $5.5 \pm 1.1$ vs. MO: $5.7 \pm 0.4, P=0.566$ ). In contrast, we found significantly lower levels in SS and NASH subgroups compared with NL subjects (NL: $7.3 \pm 0.5$; SS: $5.6 \pm 0.7, P=0.045$; NASH: $4.9 \pm 0.5, P=$ $0.017)$. Interestingly, RBP4 and adiponectin expression in VAT from MO were strongly correlated $(r=0.401, P=0.025)$.

RBP4 mRNA liver expression. The analysis of RBP4 hepatic expression showed similar results in the controls to those in $\mathrm{MO}$ $(P=0.27)$ (Figure 1c). Within MO women, we did not find any differences in the expression of RBP4 between NL vs. SS and NASH groups ( $P=0.73$ and $P=0.41$, respectively) (Figure 1f). Interestingly, NASH-mild showed higher RBP4 expression than $\mathrm{NASH}$-moderate/severe $(7.83 \pm 0.91$ vs. $5.02 \pm 0.8, P=0.038)$.

Liver adiponectin expression was lower in $\mathrm{MO}$ compared with controls (C: $2.7 \times 10^{-4} \pm 1.1 \times 10^{-4}$ vs. MO: $5 \times 10^{-5} \pm 3 \times 10^{-5}$,
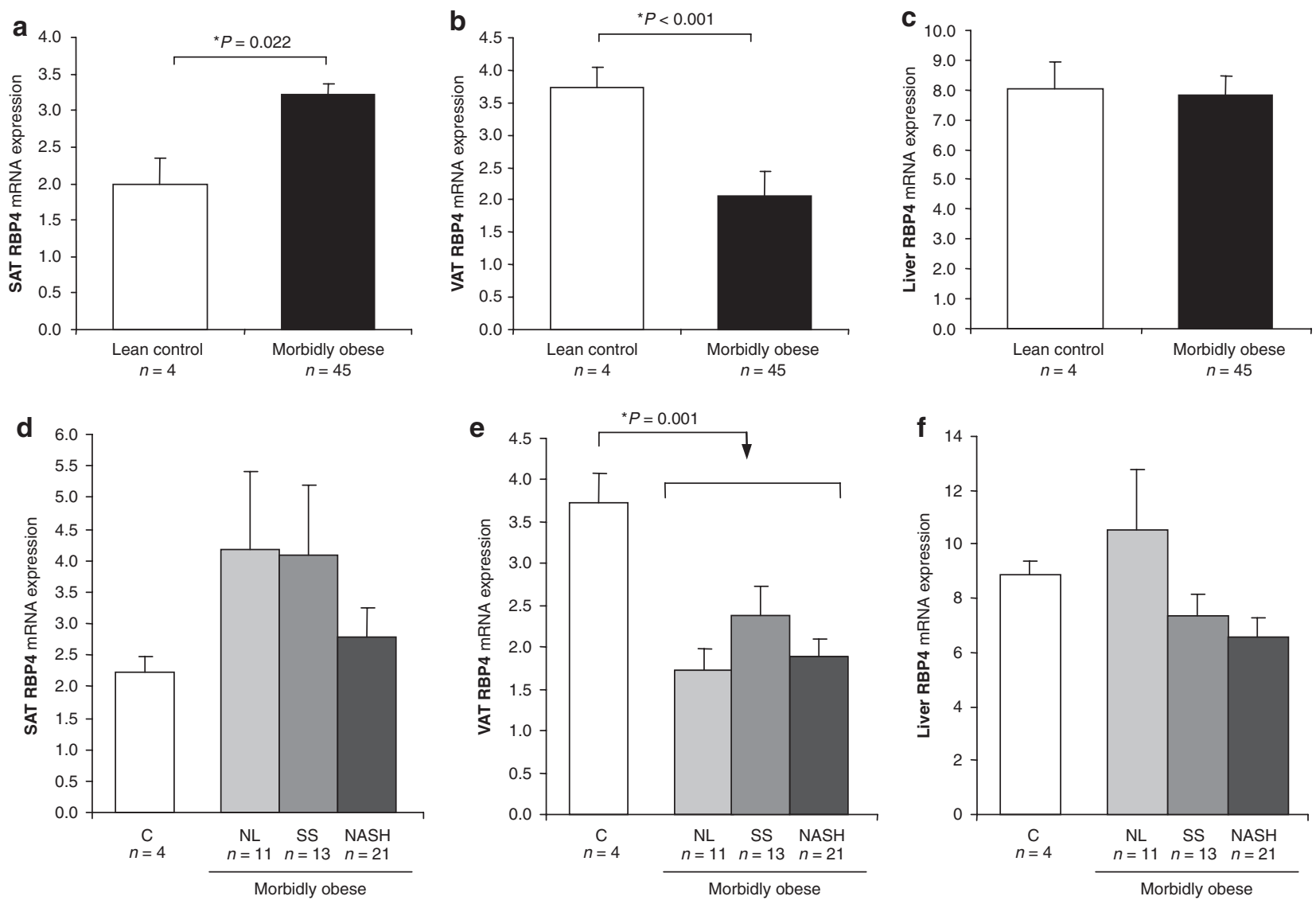

Figure 1 RBP4 gene expression in (a,d) subcutaneous and (b,e) visceral adipose tissues, and in (c,f) liver respectively. ${ }^{*}$ Indicates statistically significant differences between groups $(P<0.05)$. C, lean controls with normal liver histology $(n=4)$; mRNA, messenger RNA; NASH, morbidly obese women with nonalcoholic steatohepatitis $(n=21)$; NL, morbidly obese women with normal liver histology; SAT, subcutaneous adipose tissue; SS, morbidly obese women with simple steatosis $(n=13)$; VAT, visceral adipose tissue. 
$P=0.029)$. MO subgroups displayed similar adiponectin mRNA levels (data not shown). In liver, RBP4 and adiponectin expression were strongly correlated $(r=0.530, P=0.001)$ in the MO cohort.

Multivariate linear regression analysis to predict RBP4 expression in tissues. We performed a multivariate linear regression analysis to identify the best predictors of liver, subcutaneous, and visceral RBP4 mRNA expression in the MO cohort. We evaluated the effect of metabolic variables on the varaibility of the RBP4 expression levels in each tissue analyzed. The variables entered on the analysis were: fasting glucose, insulin, BMI, triglycerides, high-density lipoprotein, and total cholesterol. The results indicated that the variables included were not significant predictors of the RBP4 expression in any of tissues studied (data not shown).

We then evaluated the effect of the mRNA expression of other adipocytokines on the varaibility of the RBP4 expression. The results now indicated that among the adipocytokines included in the model (adiponectin, TNF- $\alpha$, and resistin), adiponectin is the one with the highest effect on the variability of RBP4 expression in the last step (3rd) of the backward exclusion method in VAT and liver.

In VAT the results were: $\beta=0.351,95 \%$ CI $0.074-0.628, P=$ 0.015 . In this model, adiponectin expression explained a $29 \%$ of the variability RBP4 expression (Nagelkerke $R^{2}=0.293$ ).

In liver the results were: $\beta=0.921,95 \%$ CI $0.404-1.437, P=$ 0.001 . In this model, adiponectin expression explained a $40 \%$ of the variability RBP4 expression (Nagelkerke $R^{2}=0.399$ ).

In contrast, the circulating levels of adiponectin, resistin or TNF- $\alpha$ were not relevant predictors of RBP4 expression (data not shown).

\section{Comparison between liver, VAT, and SAT RBP4 gene expression}

When the relative mRNA expression of RBP4 was analyzed in the three tissues, we observed that the liver had the highest expression compared with either fat depot in all the groups analyzed (Controls, SAT: $2.22 \pm 0.24$, VAT: $3.73 \pm 0.36$, liver: $8.85 \pm 0.56, P$ $=0.023$; NL, SAT: $4.17 \pm 1.24$, VAT: $1.72 \pm 0.27$, liver: $10.51 \pm 2.28$, $P=0.034$; SS, SAT: $4.09 \pm 1.12$, VAT: $2.38 \pm 0.34$, liver: $7.34 \pm 0.83$, $P=0.023$; NASH, SAT: $2.77 \pm 0.47$, VAT: $1.88 \pm 0.22$, liver: 6.57 $\pm 0.72, P=0.043)$. Thus, in the four groups studied, expression in the liver tissue was more than double the expression in either adipose tissue. In addition, in all MO groups, VAT RBP4 expression was lower than SAT expression, although these values were not statistically significant.

Circulating RBP4 levels did not correlate with its expression in any tissue analyzed ( $P$ value not significant).

\section{RBP4 expression and circulating levels according to the presence of DM}

We also compared RBP4 mRNA expression and its circulating levels in women with and without T2DM, because three women in the SS group and seven in the NASH group had T2DM.

In the SS group, the results indicate that the expression of RBP4 in SAT, VAT, and liver was not modified by the presence of diabetes (SAT: nondiabetic: $3.3 \pm 1.5$ vs. diabetic: $3.5 \pm 1.0 ; P=0.14$, VAT: nondiabetic: $2.5 \pm 1.4$ vs. diabetic:
$2.2 \pm 1.2 ; P=0.621$, liver: nondiabetic: $6.9 \pm 2.0$ vs. diabetic: $6.3 \pm 1.1 ; P=0.93$ ) and nor were its circulating levels (nondiabetic: $4.1 \pm 1.2$ vs. diabetic: $3.5 \pm 2.4 ; P=0.398$ ).

Similar results were obtained in the NASH group, subclassified according to the presence of diabetes (SAT: nondiabetic: $2.6 \pm 1.0$ vs. diabetic: $2.5 \pm 2.2 ; P=0.481$, VAT: nondiabetic: $2.5 \pm 0.9$ vs. diabetic: $1.5 \pm 0.6 ; P=0.050$, liver: nondiabetic: $6.6 \pm 2.8$ vs. diabetic: $6.5 \pm 2.4 ; P=0.900$. Circulating levels: nondiabetic: $3.0 \pm 0.5$ vs. diabetic: $3.5 \pm 1.0 ; P=0.469$ ).

\section{RBP4 expression in HepG2 cells after TNF- $\alpha$ or adiponectin exposure}

We examined RBP4 expression in vitro using HepG2 cells. Downregulation of RBP4 expression was detected $48 \mathrm{~h}$ after exposure to TNF- $\alpha$ compared to controls and to adiponectin treatment. After the exposure to adiponectin, cells expressed similar levels of RBP4 than control hepatocytes (Figure 2).

\section{DISCUSSION}

This is the first study to analyze circulating RBP4 levels together with SAT and VAT and liver gene expression in samples gathered from a cohort of MO women.

In this cohort, no differences in ALT, AST, and GGT levels were found between the MO with NL histology, SS, and $\mathrm{NASH}$. In the literature, the question of whether transaminase levels correlate with the histological findings, particularly with necroinflammatory activity and the degree of fibrosis, has still not been answered definitively (25). In clinical practice, there is so far no means of differentiation reliably between SS and NASH solely on the basis of noninvasive (e.g., laboratory chemical) diagnostic tests, i.e., without liver biopsy (26,27).

The pathogenesis of NAFLD is related to IR and, thus, it is frequently found in individuals who have central obesity or diabetes. IR and excess adiposity are associated with increased lipid

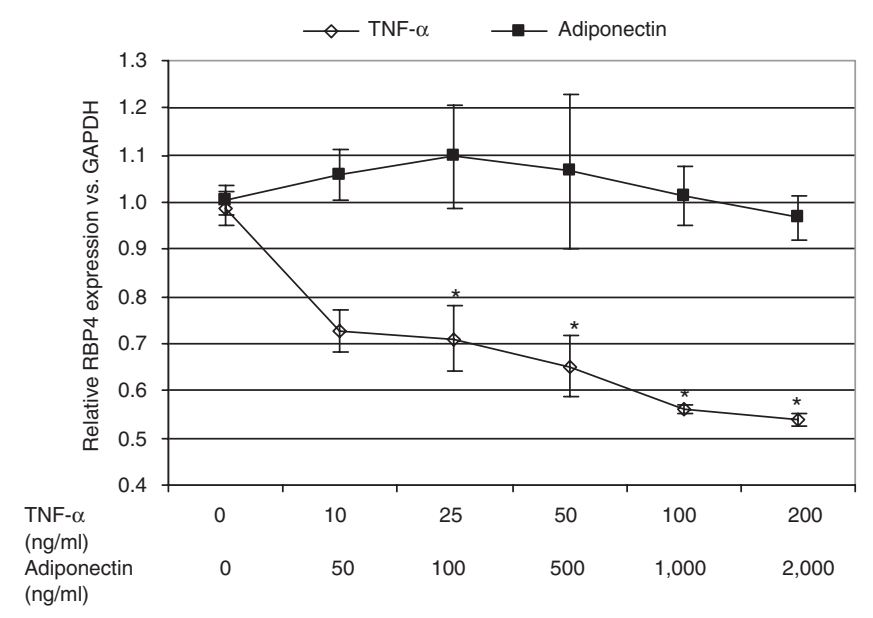

Figure 2 Determination of RBP4 gene expression in HepG2 cells after the incubation with TNF- $\alpha$ or adiponectin. Cells either untreated (control) or treated with TNF- $\alpha$ or adiponectin for $48 \mathrm{~h}$ at different concentrations. Values are mean of three independent experiments performed in triplicates. *Indicates significative $P$ values $<0.05$ vs. controls. GAPDH, glyceraldehyde 3-phosphate dehydrogenase; TNF- $\alpha$, tumor necrosis factor- $\alpha$. 


\section{ARTICLES}

\section{GENETICS}

influx into the liver and increased de novo hepatic lipogenesis, promoting hepatic triglyceride accumulation. Some authors have described that HOMA-IR was associated with increased grades of hepatic necroinflammation (28). In our study, no differences in HOMA-IR were found between the MO with NL histology, SS, and NASH. However, patients with NASH tended to present higher HOMA-IR values than MO with NL and SS. These results need to be confirmed in larger study cohorts.

Focusing on RBP4, our results indicate that its circulating levels are increased in MO women, as previously reported $(11,29)$. The relationship between systemic levels of RBP4 and triglyceride levels also confirms our previous finding in women with morbid obesity (29). This finding agrees with Seo et al. (17) and suggests a possible involvement of this adipokine with lipid metabolism. RBP4 is the sole transporter for retinol in the circulation, and retinoids are known to increase hepatic production of VLDL (30). Since triglycerides are the main component of VLDL lipoproteins, the independent association between triglycerides with RBP4 concentrations found could reflect the relationship between RBP4 and hepatic VLDL metabolism.

We then evaluated RBP4 circulating levels in relation to NAFLD histology. Interestingly, MO subjects with NL histology did not present increased levels of RBP4 compared to lean controls with a normal liver. In contrast, we found that circulating RBP4 levels were significantly elevated in MO women with NAFLD. Our observation agrees with studies suggesting that RBP4 might be related to alterations associated with the pathogenesis of NAFLD $(17,18)$. Regarding this, Stefan et al. (16) also found that circulating RBP4 correlated positively with liver fat accumulation. On the other hand, other studies reported lower or similar levels in normal weight or overweight subjects with $\operatorname{NAFLD}(19,20,31)$.

In our study, we were unable to find a relationship between RBP4 levels and IR, in agreement with other authors (17,19-21). Strikingly, we found a negative association with resistin levels, which agrees with Cengiz et al. (19). Although adipocyte-derived resistin is undisputably linked to IR in rodent models, the relevance of human resistin is complicated because it is secreted by macrophages rather than adipocytes. Recent, growing evidence indicates that human resistin is an inflammatory biomarker (32). Therefore, the negative association between RBP4 and resistin might be indicating that RBP4 has a different role, rather than being implicated directly to IR in this cohort.

The lack of association between RBP4 circulating levels and obesity and insulin-resistant states has been reviewed by Kotnik et al. (33). It was suggested that the positive associations found previously between RBP4, obesity, IR, and T2DM could be the result of decreased renal clearance of RBP4 caused by different levels of renal insufficiency in these subjects (34). In order to avoid this bias, we excluded the MO women with abnormal renal clearance from our study group. It was also proposed that the imbalance between RBP4 and retinol might be the underlying cause for the observed association between RBP4 levels and systemic insulin resistance (33).

The deregulation of adipokine expression, mainly in visceral fat depots, suggests a link between obesity and IR. Adipokines released from visceral fat in IR states associated to obesity might augment the secretion of free fatty acids into the portal circulation and thus increase their accumulation in the liver as triglycerides $(3,6,7)$. In our study, RBP4 mRNA expression in SAT and VAT appeared to be unrelated to IR or NAFLD histology. RBP4 VAT expression was significantly lower in all groups of $\mathrm{MO}$ women. Interestingly, overall visceral RBP4 expression was lower than subcutaneous expression, as recently reported (35). On the other hand, Klöting et al. (13) found that RBP4 expression was a marker of intra-abdominal fat mass, but these authors did not discriminate between normal weight and overweight/obese patients.

Using animal models, RBP4 has been described to be predominantly synthesized in liver (36), but, to our knowledge, RBP4 in liver from subjects with NAFLD has only been evaluated in a few studies (37). By immunostaining, Tönjes et al. found an increase in RBP4 according to the presence of SS and the grade of NASH (37). However, in our cohort of MO women, we found no significant relationships between RBP4 gene expression and biochemical parameters of liver function or degrees of histological liver damage. We found that the liver had the highest expression of RBP4 in all physiological states studied, but there is a lack of correlation between hepatic expression and systemic levels.

In the present study, we have addressed for the first time in humans the circulating RBP4 levels, the gene expression of RBP4 in SAT and VAT and liver in parallel, in order to analyze the potential contribution of these tissues to the levels of RBP4 in the circulation. We did not find a correlation between the expression rates in the tissues analyzed and its circulating levels. The lack of association between RBP4 expression and its serum concentration might highlight the importance of considering the differences in the total production rate of adipose tissue-derived factors due to differences in the adiposity of the groups studied $(38,39)$.

The analysis in vitro of RBP4 gene expression in hepatocytes indicates that TNF- $\alpha$ downregulates RBP4 expression. Furthermore, RBP4 expression in liver was lower in severe NASH patients. In agreement with these results, in a previous study from our research group, we observed in vitro that RBP4 expression was strongly inhibited by TNF- $\alpha$ and lipopolysaccharide in primary human monocytes differentiated into macrophages (10). Regarding this, a negative association between RBP4 and proinflammatory cytokines has been described previously in a nonobese cohort of sepsis intensive care unit patients. The authors concluded that RBP4 displayed characteristics of a negative acute phase reactant (40). Furthermore, the analysis of the multivariate analysis showed that RBP4 and adiponectin mRNA expression in liver and VAT are strongly related. Taken together, these findings, lead us to further investigate the potential anti-inflammatory role of this protein.

One of the limitations of our study is that the results cannot be extrapolated to other obesity groups, or men. They need to be confirmed using large, population-based, prospective studies that include patients of both genders with different grades of obesity. However, in this group we diagnosed NAFLD based on the analysis of liver biopsy, which is considered the "gold standard" method. 
In conclusion, our study confirms in a homogeneous cohort of MO women that RBP4 levels are increased, and shows that MO subjects with NAFLD presented higher levels of RBP4 than those with NL histology. Furthermore, we found that, compared with abdominal fat depots, the liver is the tissue that predominantly expresses RBP4 in MO women with or without NAFLD. Finally, our results indicate that VAT RBP4 expression is downregulated in morbid obesity women, independently of hepatic condition.

Taken together, the results obtained in MO women with NAFLD bring up the use of a panel of adipokines including RBP4, as noninvasive molecular biomarkers that detect the presence of fatty liver in these patients. Larger studies are necessary to confirm these hypotheses in different obesity groups and gender.

\section{ACKNOWLEDGMENTS}

This study was supported by the Ministerio de Ciencia e Innovación of the government of Spain (grant number SAF 2008-02278, to C.R.J.), the Fondo de Investigación Sanitaria (grant number PS09/01778, to T.A.), by funds from Agència de Gestió d'Ajuts Universitaris i de Recerca (AGAUR 2009 SGR 959 to C.R.J.), Grup GEMMAIR (2010PFR-URV-B2-14 to C.R.J.), and by the Fundación Biociencia. We are grateful to Antonio Garcia-España and Pilar Budesca for their assistance in writing the manuscript.

\section{DISCLOSURE}

The authors declared no conflict of interest.

See the online ICMJE Conflict of Interest Forms for this article.

(C) 2012 The Obesity Society

\section{REFERENCES}

1. Angulo P. Nonalcoholic fatty liver disease. N Engl J Med 2002;346:1221-1231.

2. Marchesini G, Bugianesi E, Forlani G et al. Nonalcoholic fatty liver, steatohepatitis, and the metabolic syndrome. Hepatology 2003:37:917-923.

3. Smith BW, Adams LA. Non-alcoholic fatty liver disease. Crit Rev Clin Lab Sci 2011;48:97-113.

4. Fabbrini E, Sullivan S, Klein S. Obesity and nonalcoholic fatty liver disease: biochemical, metabolic, and clinical implications. Hepatology 2010;51:679-689.

5. Karastergiou K, Mohamed-Ali V. The autocrine and paracrine roles of adipokines. Mol Cell Endocrinol 2010;318:69-78.

6. Gnacinska M, Malgorzewicz S, Stojek M, Lysiak-Szydlowska W, Sworczak K. Role of adipokines in complications related to obesity: a review. Adv Med Sci 2009;54:150-157.

7. Polyzos SA, Kountouras J, Zavos C. Nonalcoholic fatty liver disease: the pathogenetic roles of insulin resistance and adipocytokines. Curr Mol Med 2009;9:299-314.

8. Tsutsumi C, Okuno M, Tannous L et al. Retinoids and retinoid-binding protein expression in rat adipocytes. J Biol Chem 1992;267:1805-1810.

9. Quadro L, Blaner WS, Hamberger L et al. The role of extrahepatic retinol binding protein in the mobilization of retinoid stores. J Lipid Res 2004;45:1975-1982.

10. Broch M, Ramírez R, Auguet MT et al. Macrophages are novel sites of expression and regulation of retinol binding protein-4 (RBP4). Physiol Res 2010;59:299-303.

11. Graham TE, Yang Q, Blüher M et al. Retinol-binding protein 4 and insulin resistance in lean, obese, and diabetic subjects. N Engl J Med 2006;354:2552-2563.

12. Cho YM, Youn BS, Lee H et al. Plasma retinol-binding protein-4 concentrations are elevated in human subjects with impaired glucose tolerance and type 2 diabetes. Diabetes Care 2006;29:2457-2461.

13. Klöting N, Graham TE, Berndt J et al. Serum retinol-binding protein is more highly expressed in visceral than in subcutaneous adipose tissue and is a marker of intra-abdominal fat mass. Cell Metab 2007;6:79-87.

14. Janke J, Engeli S, Boschmann M et al. Retinol-binding protein 4 in human obesity. Diabetes 2006;55:2805-2810.

15. Broch M, Auguet MT, Ramírez R et al. Parallel downregulation of retinol-binding protein-4 and adiponectin expression in subcutaneous adipose tissue of non-morbidly obese subjects. Eur J Endocrinol 2009;161:87-94.

16. Stefan N, Hennige AM, Staiger $\mathrm{H}$ et al. High circulating retinol-binding protein 4 is associated with elevated liver fat but not with total, subcutaneous, visceral, or intramyocellular fat in humans. Diabetes Care 2007;30:1173-1178.

17. Seo JA, Kim NH, Park SY et al. Serum retinol-binding protein 4 levels are elevated in non-alcoholic fatty liver disease. Clin Endocrinol (Oxf) 2008;68:555-560

18. Wu H, Jia W, Bao Y et al. Serum retinol binding protein 4 and nonalcoholic fatty liver disease in patients with type 2 diabetes mellitus. Diabetes Res Clin Pract 2008;79:185-190.

19. Cengiz C, Ardicoglu Y, Bulut S, Boyacioglu S. Serum retinol-binding protein 4 in patients with nonalcoholic fatty liver disease: does it have a significant impact on pathogenesis? Eur J Gastroenterol Hepatol 2010;22:813-819.

20. Schina M, Koskinas J, Tiniakos D et al. Circulating and liver tissue levels of retinol-binding protein-4 in non-alcoholic fatty liver disease. Hepatol Res 2009;39:972-978

21. Alkhouri N, Lopez R, Berk M, Feldstein AE. Serum retinol-binding protein 4 levels in patients with nonalcoholic fatty liver disease. J Clin Gastroenterol 2009;43:985-989.

22. Brunt EM, Janney CG, Di Bisceglie AM, Neuschwander-Tetri BA, Bacon BR. Nonalcoholic steatohepatitis: a proposal for grading and staging the histological lesions. Am J Gastroenterol 1999;94:2467-2474.

23. Kleiner DE, Brunt EM, Van Natta M et al. Design and validation of a histological scoring system for nonalcoholic fatty liver disease. Hepatology 2005;41:1313-1321.

24. WHO. Definition and diagnosis of diabetes mellitus and intermediate hyperglycemia. WHO 2006:1-46.

25. Sonsuz A, Basaranoglu M, Ozbay G. Relationship between aminotransferase levels and histopathological findings in patients with nonalcoholic steatohepatitis. Am J Gastroenterol 2000;95:1370-1371.

26. Farrell GC, Larter CZ. Nonalcoholic fatty liver disease: from steatosis to cirrhosis. Hepatology 2006;43:S99-S112.

27. Bellentani S, Bedogni G, Miglioli L, Tiribelli C. The epidemiology of fatty liver. Eur J Gastroenterol Hepatol 2004;16:1087-1093.

28. Hui JM, Hodge A, Farrell GC et al. Beyond insulin resistance in NASH: TNF-alpha or adiponectin? Hepatology 2004;40:46-54.

29. Broch M, Gómez JM, Auguet MT et al. Association of retinol-binding protein-4 (RBP4) with lipid parameters in obese women. Obes Surg 2010;20:1258-1264.

30. Gerber LE, Erdman JW Jr. Retinoic acid and hypertriglyceridemia. Ann N Y Acad Sci 1981;359:391-392.

31. Milner KL, van der Poorten D, Xu A et al. Adipocyte fatty acid binding protein levels relate to inflammation and fibrosis in nonalcoholic fatty liver disease. Hepatology 2009;49:1926-1934.

32. Schwartz DR, Lazar MA. Human resistin: found in translation from mouse to man. Trends Endocrinol Metab 2011;22:259-265.

33. Kotnik P, Fischer-Posovszky P, Wabitsch M. RBP4: a controversial adipokine. Eur J Endocrinol 2011;165:703-711.

34. Chang $\mathrm{YH}$, Lin KD, Wang CL et al. Elevated serum retinol-binding protein 4 concentrations are associated with renal dysfunction and uric acid in type 2 diabetic patients. Diabetes Metab Res Rev 2008;24:629-634.

35. Kos K, Wong S, Tan BK et al. Human RBP4 adipose tissue expression is gender specific and influenced by leptin. Clin Endocrinol (Oxf) 2011;74:197-205.

36. Yang $Q$, Graham TE, Mody N et al. Serum retinol binding protein 4 contributes to insulin resistance in obesity and type 2 diabetes. Nature 2005;436:356-362.

37. Tönjes A, Blüher M, Stumvoll M. Retinol-binding protein 4 and new adipocytokines in nonalcoholic fatty liver disease. Curr Pharm Des 2010;16:1921-1928.

38. Barth S, Klein P, Horbach T et al. Expression of neuropeptide Y, omentin and visfatin in visceral and subcutaneous adipose tissues in humans: relation to endocrine and clinical parameters. Obes Facts 2010;3:245-251.

39. Terra X, Quintero Y, Auguet T et al. FABP 4 is associated with inflammatory markers and metabolic syndrome in morbidly obese women. Eur $J$ Endocrinol 2011;164:539-547.

40. Koch A, Weiskirchen R, Sanson E et al. Circulating retinol binding protein 4 in critically ill patients before specific treatment: prognostic impact and correlation with organ function, metabolism and inflammation. Crit Care 2010;14:R179. 